[RADiocarbon, Vol.. 21, No. 1, 1979, P. 107-112]

\title{
UNIVERSITY OF MIAMI RADIOCARBON DATES XIV
}

\author{
P M CALVERT, D S INTRONE and J J STIPP \\ Department of Geology, University of Miami \\ Coral Gables, Florida 33124
}

The following radiocarbon dates are a partial list of samples measured since January 1978 . The chemical, counting and calculation procedures are the same as indicated in R, v 20, p 000-000.

\section{SAMPLE DESCIRIPTIONS}

I. ARCHAEOLOGIC SAMPLES

\section{Bimini Atlantis series}

\section{A. Bahamas}

Carbonate rock samples, postulated rd bldg material of lost civilization Atlantis (Harrison, 1971; Shinn, 1977) dated as whole rock and in separated components of shell material and cement, to determine maximum age of "road-like" formation. Cores from blocks $-4.6 \mathrm{~m}$, oriented parallel to and ca $10 \mathrm{~km}$ from $\mathrm{W}$ Bimini shoreline $\left(26^{\circ}\left(0^{\prime} \mathrm{N}, 77^{\circ} 30^{\prime} \mathrm{W}\right)\right.$. Coll 1977 by E Shinn, USGS, Fisher Island Sta, Miami, Florida, and subm 1978 by D Drevitson, Univ Miami. Samples id by depth in core.

$\begin{array}{lll}\text { UM-1359. } & \text { F ( a ) } 4 \text { to } 6 \mathrm{~cm} \text {, whole rock } & 2780 \pm 70 \\ \text { UM-1360. } & \text { F (b) } 15.5 \text { to } 17 \mathrm{~cm} \text {, whole rock } & 3500 \pm 80 \\ \text { UM-1361. } & \text { F (c) } 27.5 \text { to } 29 \mathrm{~cm} \text {, whole rock } & 3350 \pm 90 \\ \text { UM-1362. } & \text { H, shells } & 3510 \pm 70 \\ \text { UM-1363. } & \text { B } 0 \text { to } 5 \mathrm{~cm}, \text { cement } & 2750 \pm 80 \\ \text { UM-1364. } & \text { B } 12.9 \text { to } 16.8 \mathrm{~cm}, \text { cement } & 2770 \pm 80 \\ \text { UM-1365. } & \text { B } 24.7 \text { to } 29.8 \mathrm{~cm}, \text { cement } & 2840 \pm 70\end{array}$

\section{Granada series}

\section{B. United States}

Shell and charcoal from prehistoric Indian midden at mouth of Miami R on N bank (25 46 $36^{\prime \prime}$ N, 80 $10^{\circ} 12^{\prime \prime}$ W) dated site. Coll 1978 by C Martinez, Bur Hist Sites \& Properties, Tallahassee, Florida and subm by J Mattes, Univ Miami. Comment (P Calvert): external and internal portions of shells (strombus gigas) processed separately in conjunction with x-ray defraction analysis to study effects of possible recrystallization in samples.

\section{UM-1366. FS-198-Ch}

$1740 \pm 90$

Charcoal from 60 to $75 \mathrm{~cm}$ below ground surface.

$$
\delta^{13} \mathbf{C}=-\mathbf{2 5 . 5 6} / \%
$$

UM-1367. Duplicate run UM-1366

$$
1260 \pm 70
$$


UM-1368. FS-198-sh

$\delta^{13} \mathbf{C}=+1.41 \%$

Internal portion of shell found with UM-1366 and -136760 to $75 \mathrm{~cm}$ below ground surface.

UM-1369. External portion UM-1368

$$
\begin{array}{r}
1590 \pm 90 \\
\delta^{13} \mathbf{C}=+\mathbf{1 . 8 9} \% \\
1490 \pm 250 \\
1380 \pm 100 \\
1720 \pm 70 \\
\delta^{13} \mathbf{C}=-\mathbf{0 . 6 6} \%
\end{array}
$$

UM-1371. Duplicate run UM-1369

UM-1372. Duplicate run UM-1368

UM-1373. FS-87-sh

External portion of shell 135 to $150 \mathrm{~cm}$ below ground surface.

UM-1374. Internal portion UM-1373

$$
\begin{array}{r}
1870 \pm 90 \\
\delta^{13} \mathrm{C}=-0.54 \% \\
1780 \pm 100 \\
\delta^{13} \mathrm{C}=-\mathbf{2 5 . 5 3} \%
\end{array}
$$

UM-1370. FS-87-Ch surface.

Charcoal found with UN-1373 and $-1374,135$ to $150 \mathrm{~cm}$ below ground

\section{Kenan Field series}

Three carbonized wood samples and one carbonized marine shell sample from Kenan Field, Sapelo I., Georgia (NGR 473500E, 348000N). Coll 1977 by M R Crook, Florida State Mus and subm 1978 by M R Crook and J T Milanich, Florida State Mus, Gainesville, Florida.

UM-1388. FOA3 r (49)

$$
\begin{aligned}
& 970 \pm 60 \\
\delta^{13} \mathbf{C}= & -\mathbf{2 5 . 6 0} / / 0
\end{aligned}
$$

Carbonized wood from probable earth oven that was later used as refuse pit.

UM-1389. Fea. 219 (51)

$$
\begin{array}{r}
\mathbf{1 6 6 0} \pm \mathbf{7 0} \\
\delta^{13} \mathrm{C}=-\mathbf{2 5 . 5 2} \% \text { \% }
\end{array}
$$

Carbonized wood from hearth that preceded construction of wall trench assoc with Structure 2.

UM-1390. FEA 108 ( 53 )

$$
790 \pm 70
$$

Carbonized oyster shell from hearth assoc with Structure 1. Sample is from uppermost undisturbed level $25 \mathrm{~cm}$ below surface.

UM-1391. FEA 109 (54)

$$
\begin{aligned}
& 1200 \pm 60 \\
& \delta^{13} \mathbf{C}=-\mathbf{2 6 . 6 4} \%
\end{aligned}
$$

Carbonized wood from a probable post hole assoc with construction of Structure 1 .

\section{Kings Bay series}

Several carbonized wood and oyster samples from Feature 2 and Feature 4 pits located at King's Bay Naval facility, Comden Co, Georgia 
(NGR $340600 \mathrm{mN}, 451000 \mathrm{mE}$ ). Coll and subm 1978 by R Johnson, Univ Florida, Gainesville. Comment (DI): UM-1429 and -1430 are from Feature 4, a trash pit, ca 60 to $62 \mathrm{~cm}$ below ground surface. UM-1431, -1433 are from Feature 2, hearth $24 \mathrm{~cm}$ below present ground surface assoc with numerous sherds of fiber-tempered ceramic vessel(s).

UM-1429. Fea. 4 FS \#116, KBS 12

Carbonized wood.

UM-1430. Fea. 4 FS \#116a, KBS 12 Oyster shell.

UM-1431. Fea. 2 FS \#62, KBS 12 Oyster shell.

UM-1432. Fea. 2 FS \# 56, KBS 8

Carbonized wood.

UM-1433. Fea. 2 FS \#60, KBS 8

Carbonized wood.
$2330 \pm 180$

$\delta^{13} \mathbf{C}=-\mathbf{2 6 . 8 9} \%$ 。

$$
980 \pm 70
$$$$
1330 \pm 60
$$

$5000 \pm 180$

$\delta^{13} \mathbf{C}=-\mathbf{2 7 . 9 9} / c^{\circ}$

$$
4260 \pm 100
$$

$\delta^{13} \mathbf{C}=-\mathbf{2 5 . 5 4} \%$ 。

\section{Welborn series}

Three charred Pinus samples from Florida Archeol Site 8 Co 17 in W Columbia Cio, Florida, ca $4 \mathrm{~km}$ E of Welborn, Florida $\left(30^{\circ} 13^{\prime} 26^{\prime \prime} \mathrm{N}\right.$, $\left.82^{\circ} 46^{\prime} 15^{\prime \prime} \mathrm{W}\right)$. Coll and subm 1978 by J T Milanich.

UM-1434. FS 956-A

$$
\delta^{13} \mathrm{C}=-\mathbf{1 4 6 0} \pm 70.04 \%
$$
Florida.

Dates Mound $\mathrm{C}$ and Kolomoki-style pedestaled effigy vessels in $\mathrm{N}$

UM-1435. FS 1094-A

$$
\delta^{13} \mathrm{C}=-\mathbf{1 7 2 0} \pm \mathbf{8 0} .80
$$
at site.

Dates Mound A and late proto-chiefdom level of social organization

UM-1436. FS 1022-A

$$
1470 \pm 70
$$

Dating required for clarification of Mound $\mathrm{C}$ position.

\section{UM-1395. Chaco River}

$$
\begin{array}{r}
1910 \pm 90 \\
\delta^{13} \mathrm{C}=-23.92 \%
\end{array}
$$

Carbonized wood from Late Basketmaker III pithouse site on Chaco $\mathrm{R}$ in NW New Mexico (36 $\left.07^{\prime} 03^{\prime \prime} \mathrm{N}, 108^{\circ} 11^{\prime} 12^{\prime \prime} \mathrm{W}\right)$. Material taken from undisturbed hearth ca $2 \mathrm{~m}$ below site surface. Coll and subm 1977 by W H Doleman, Mus New Mexico, Santa Fe. 


\section{E Pacific Rise series}

II. GEOLOGIC SAMPLES

A. SE Pacific

Foram and diatom ooze from E Pacific Rise and Bellinghausen abyssal plain, dated to study sedimentation rate since last glaciation. All samples from SE flank of E Pacific Rise unless stated otherwise and id by depth in core. Coll 1978 by M Dinkelman, Florida State Univ, Tallahassee, and subm 1978 by P Sahler, Univ Miami.

UM-1375. E17-30, 36 to $38 \mathrm{~cm}$

$15,730 \pm 580$

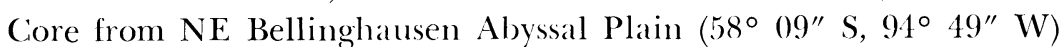
from water depth $627 \mathrm{~m}$.

UM-1376. PC-E-2, 20 to $22 \mathrm{~cm}$

$6560 \pm 250$

Core $\left(56^{\circ} 3^{\prime} 30^{\prime \prime} \mathrm{S}, 115^{\circ} 3^{\prime} 42^{\prime \prime} \mathrm{W}\right)$, water depth $518 \mathrm{~m}$.

UM-1377. PCE32-22, 13 to $15 \mathrm{~cm}$

$6920 \pm 100$

Core $\left(54^{\circ} 56^{\prime} 12^{\prime \prime} \mathrm{S}, 120^{\circ} 00^{\prime} \mathrm{W}\right)$, water depth $457 \mathrm{~m}$.

UM-1378. PCE33-22, 27 to $29 \mathrm{~cm}$

$8380 \pm 200$

Core $\left(54^{\circ} 56^{\prime} 12^{\prime \prime} \mathrm{S}, 120^{\circ} 00^{\prime} \mathrm{W}\right)$, water depth $457 \mathrm{~m}$.

UM-1379. PCE33-22, 66 to $69 \mathrm{~cm}$

$21,370 \pm 420$

Core $\left(54^{\circ} 56^{\prime} 12^{\prime \prime} \mathrm{S}, 120^{\circ}\left(00^{\prime} \mathrm{W}\right)\right.$, water depth $457 \mathrm{~m}$

UM-1380. PCE11-2, 45 to $47 \mathrm{~cm} \quad 10,010 \pm 380$

Core $\left(56^{\circ} 03^{\prime} 30^{\prime \prime} \mathrm{S}, 115^{\circ} 03^{\prime} 42^{\prime \prime} \mathrm{W}\right)$, water depth $518 \mathrm{~m}$.

UM-1381. PCE11-3, 18 to $20 \mathrm{~cm} \quad 9570 \pm 300$

Core $\left(56^{\circ} 54^{\prime} 12^{\prime \prime} \mathrm{S}, 115^{\circ} 14^{\prime} 36^{\prime \prime} \mathrm{W}\right)$, water depth $671 \mathrm{~m}$.

\section{Surinam series}

\section{B. South America}

Shell, shell fragment and peat debris from Surinam. Dated to reconstruct Holocene depositional sequence on shoreline. Coll 1977 and subm 1978 by J Rine, Univ Miami, Fisher Island, Miami Beach, Florida.

UM-1406. S-XI : 1139 to $1264 \mathrm{~cm}$

$7060 \pm 100$ $\delta^{13} \mathrm{C}=-27.95 \% / c$

Loose peat debris from peaty mud $\left(55^{\circ} 30^{\prime} 15^{\prime \prime} \mathrm{W}, 05^{\circ} 49^{\prime} 00^{\prime \prime} \mathrm{N}\right)$. Coll from core $15 \mathrm{~km}$ from present shoreline.

UM-1407. S-Transect: Beach

$109.0 \% \bmod$

Chione, Crassotrea from active beach (55 $\left.31^{\prime} 30^{\prime \prime} \mathrm{W}, 05^{\circ} 59^{\prime} 40^{\prime \prime} \mathrm{N}\right)$.

UM-1408. P-13: 50 to $95 \mathrm{~cm}$

$2320 \pm 80$

Core containing bivalves from near base of relict beach ridge $\left(55^{\circ}\right.$ $\left.12^{\prime} 30^{\prime \prime} \mathrm{W}, 05^{\circ} 51^{\prime} 30^{\prime \prime} \mathrm{N}\right)$. 
UM-1409. P-10: 200 to $230 \mathrm{~cm}$

$1730 \pm 120$

Core containing Chione, Tellin and unknown shell fragments from base of quartz sand beach sequence on relict beach ridge $\left(55^{\circ} 11^{\prime} 00^{\prime \prime} \mathrm{W}\right.$, $\left.05^{\circ} 53^{\prime} 10^{\prime \prime} \mathrm{N}\right)$.

\section{Card Sound series}

\section{United States}

Peat samples from $30 \mathrm{~m}$ wide fringing red mangrove stand along Card Sound $\left(25^{\circ} 20^{\prime} 35^{\prime \prime} \mathrm{N}, 80^{\circ} 20^{\prime} 00^{\prime \prime} \mathrm{W}\right)$, Florida as part of study (R, v 20, no. 3, p 511-512) of effects of peat deposits on underlying limestone. Sample id by depth below ground surface. Coll 1978 by J Meeder, D Introne, and V Skinner, Univ Miami and subm by V Skinner.

UM-1339. Core-1, 19 to $25 \mathrm{~cm}$

$$
\begin{aligned}
& 1630 \pm 70 \\
& \delta^{13} \mathbf{C}=-\mathbf{2 5 . 0 2} / \% \\
& 4830 \pm 90 \\
& \delta^{13} \mathbf{C}=-\mathbf{2 5 . 1 8} / / 1 \\
& 900 \pm 70 \\
& \delta^{13} \mathbf{C}=-24.71 \% \\
& 4830 \pm 70 \\
& \delta^{13} \mathrm{C}=-26.12 \% \\
& 500 \pm 60 \\
& 3300 \pm 60 \\
& 5240 \pm 80 \\
& \delta^{13} \mathrm{C}=-\mathbf{2 6 . 5 2} / \%
\end{aligned}
$$

UM-1340. Core-1, 172 to $187 \mathrm{~cm}$

UM-1341. Core-2, 28 to $36 \mathrm{~cm}$

UM-1342. Core-2, 141 to $147 \mathrm{em}$

UM-1343. Core-3, 26 to $32 \mathrm{~cm}$

UM-1344. Core-3, 84 to $90 \mathrm{~cm}$

\section{UM-1345. Core-3, 165 to $173 \mathrm{~cm}$}

\section{Oak Island series}

Peat and wood samples dated to study ancient lagoonal environment on North Carolina shoreline, Oak I. (39० $\left.53^{\prime} 30^{\prime \prime} \mathrm{N}, 78^{\circ} 00^{\prime} 07^{\prime \prime} \mathrm{W}\right)$. Coll and subm 1978 by W Cleary, Univ North Carolina, Wilmington.

\section{UM-1354. Oak Island-1}

$$
\begin{array}{r}
1440 \pm 70 \\
\delta^{13} \mathrm{C}=-\mathbf{2 6 . 8 2} / \%
\end{array}
$$

Peat from exposed face on Yawpow Beach near high tide line.

\section{UM-1355. Oak Island-2}

$$
\delta^{13} \mathrm{C}=\begin{array}{r}
\mathbf{5 0 0} \pm \mathbf{6 0} \\
-\mathbf{2 8 . 1 1} 1 / \%
\end{array}
$$

Peat from interticlal zone of beach face below Long Beach pond.

UM-1356. Oak Island-3

$$
1300 \pm 70
$$

Peat from within Long Beach pond.

$$
\delta^{13} \mathbf{C}=-\mathbf{2 6 . 8 7} / / c
$$


UM-1357. Oak Island-4

$1410 \pm 80$

Wood from stump forest Caswell beach.

\section{Caribbean}

\section{UM-1394. Green Cay Boring}

$4050 \pm 90$

Coral boring taken $25 \mathrm{~m}$ below sea level from Tongue of Ocean in vicinity of Green Cay, Bahamas $\left(24^{\circ} 04^{\prime} \mathrm{N}, 77^{\circ} 10^{\prime} \mathrm{W}\right)$. Sample taken to calibrate facies anatomy on leeward margin of carbonate platform. Coll 1976 by drilling contractor for Univ Miami, Fisher I., Miami Beach, Florida.

\section{RFFFRENCFS}

Calvert, M, Introne, D, and Stipp, J J, 1978, University of Miami radiocarbon dates XIII: Radiocarbon, v 20, p. 000-000.

Harrison, W, 1971, Atlantis Undiscovered-Bimini, Bahamas: Nature, v 230, p 287-289. Shinn, E A, 1977, Atlantis Hoax?, unpub paper, p 1-11. 